\title{
Vapor-Phase Transport Deposition, Characterization, and Applications of Large Nanographenes
}

\author{
Ahmad N. Abbas, ${ }^{\ddagger, \|}$ Bilu Liu, ${ }^{\ddagger}$ Akimitsu Narita, ${ }^{\S}$ Lukas F. Dössel, ${ }^{\S}$ Bo Yang, ${ }^{\S}$ Wen Zhang, ${ }^{\S}$ Jianshi Tang, ${ }^{\dagger}$ \\ Kang L. Wang, ${ }^{\dagger}$ Hans Joachim Räder, ${ }^{\S}$ Xinliang Feng, ${ }^{\S}$ Klaus Müllen, ${ }^{\S}$ and Chongwu Zhou* ${ }^{\ddagger}$ \\ ${ }^{\ddagger}$ Department of Electrical Engineering, University of Southern California, Los Angeles, California 90089, United States \\ ${ }^{\S}$ Max Planck Institute of Polymer Research, Ackermannweg 10, 55128 Mainz, Germany \\ ${ }^{\dagger}$ Device Research Laboratory, Department of Electrical Engineering, University of California, Los Angeles, California 90095, United \\ States \\ "Department of Electrical Engineering, University of Jeddah, Abdullah Sulayman St, Jeddah 22254, Saudi Arabia
}

\section{Supporting Information}

ABSTRACT: Recently, chemical synthesis of a range of large nanographene molecules with various shapes and sizes opened a new path to utilize them in various applications and devices. However, due to their extended aromatic cores and high molecular weight, film formation of large nanographene molecules, bearing more than $90 \mathrm{sp}^{2}$ carbon atoms in aromatic cores, is very challenging, which has prevented their applications such as in thin-film transistors. Here, we developed an effective approach to prepare films of such large nanographene molecules using a vapor-phase transport (VPT)
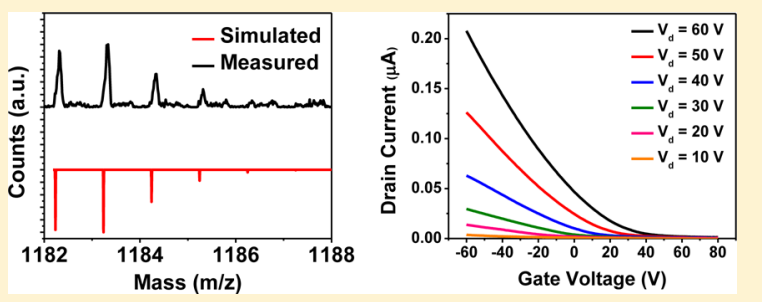
technique based on molecule sublimation. The VPT of these molecules was made possible by combining the molecules and the target substrate in a small confinement of vacuum-sealed glass tube, so that a small amount of sublimation can be utilized to create films. Surprisingly, such heavy and large molecules can be deposited on any substrate by this method to create films of assembled large nanographene molecules while maintaining their aromatic cores intact, which was confirmed using mass spectrometry measurements. Moreover, field-effect transistors based on these films are depleted and show significantly improved current on/off ratio compared to previous large nanographene-based transistors fabricated using liquid-phase-based process. Our work shows that VPT deposition can be a viable technique to prepare films based on large nanographene molecules and potentially other high molecular weight compounds, which may find exciting applications in electronics and optoelectronics.

\section{INTRODUCTION}

Advances in organic chemistry have allowed the bottom-up synthesis of graphene nanostructures such as graphene nanoribbons (GNRs) with various lengths and edge structures ${ }^{1-6}$ as well as large polycyclic aromatic hydrocarbons (PAHs), namely nanographenes. ${ }^{7-19}$ This progress has provided a high degree of design and tunability to apply such chemically synthesized structures to diverse applications. Specifically, large nanographenes, which we define here as structurally defined PAHs possessing more than $90 \mathrm{sp}^{2}$ carbon atoms in the aromatic core, are highly promising with their extended aromatic cores, which lower their bandgaps and predicted to enhance their intrinsic charge-carrier mobilities. ${ }^{13}$ The processing and deposition of the nanographenes into films on substrates in a reliable and controllable manner without destroying their structure are of extreme importance to the development of various applications utilizing such large molecules and yet have proven to be challenging. So far, liquid-phase processing is the most commonly used method to prepare films of nanographenes. ${ }^{1,4,5,20}$ Liquid-phase process starts with dispersing nanographene molecules in a solvent, followed by drop-casting, spin-coating, zone-casting, ${ }^{21}$ or incubating the dispersion on top of a target substrate. However, processing of large nanographenes in liquid phase is difficult due to their low solubility in solvents and their tendency to stack and form aggregates in the solution. ${ }^{1,3,20,22}$ To the best of our knowledge, there is hitherto no report on field-effect transistors (FETs) of large nanographenes, except for several studies on chemically synthesized GNRs, ${ }^{4,5,20}$ which are regarded as large nanographenes in our definition. In addition, liquid-phase processing of such GNRs has so far yielded FETs with rather low current on/off ratios only up to $\sim 20 .{ }^{4,5,20}$ Besides liquid-phase processing, another possible approach would be vacuum sublimation. However, it has been perceived that thermal cracking prevents the deposition of large nanographene through sublimation. ${ }^{22}$ We have therefore developed another deposition method of nanographenes, using soft-landing mass spectrometry, but this method cannot supply enough material for device fabrication. ${ }^{22}$ Consequently, a reliable method to prepare films usable in electronic, optical, and sensing applications is highly desired for the fundamental study of the

Received: January 9, 2015

Published: March 31, 2015 
a

C
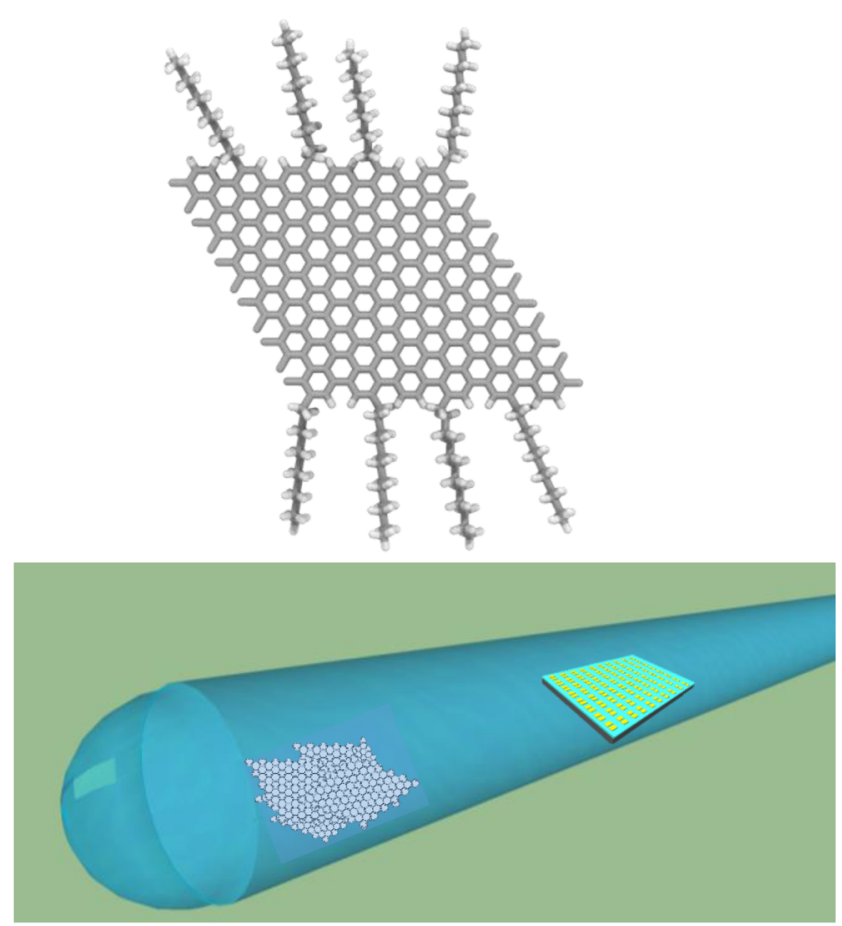

e

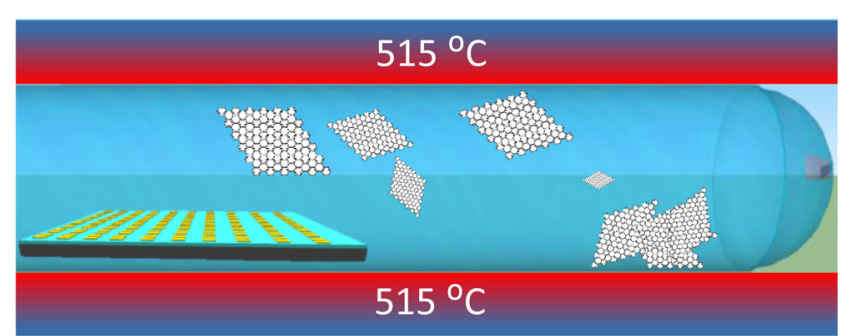

f

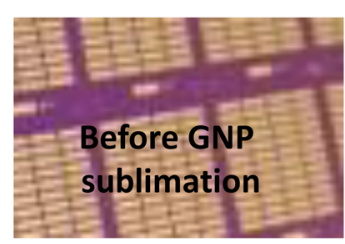

g

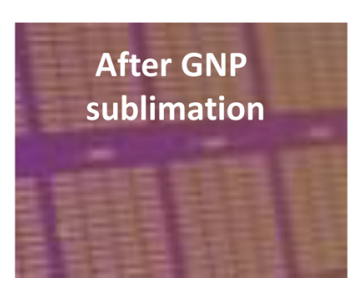

h

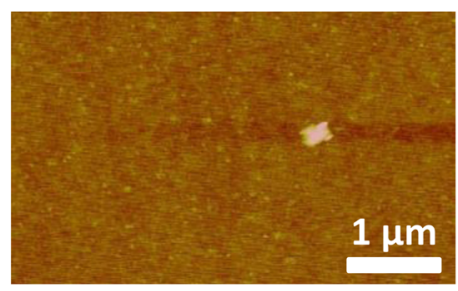

i

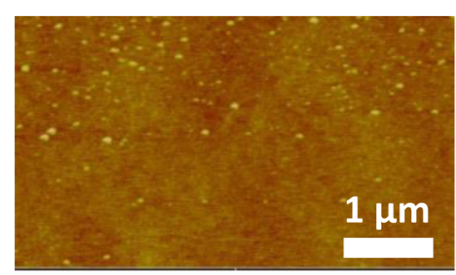

b

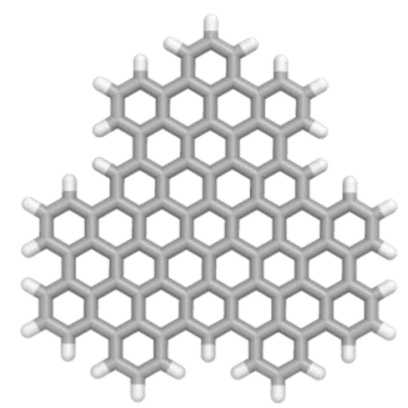

d

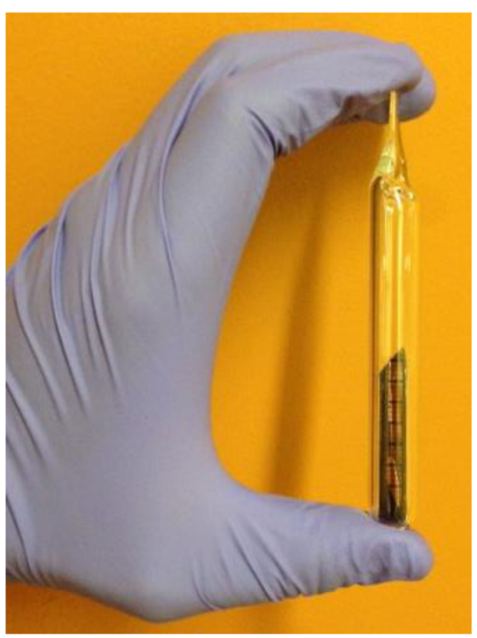

Figure 1. Molecule structure, VPT deposition, and characterization of the nanographene molecules. (a) Molecule structure of GNP (dangling bonds at edges indicate longitudinal repeat of such structure). (b) Molecular structure of $\mathrm{C}_{96}$ molecules used in this study. Carbon and hydrogen atoms are represented in gray and white, respectively. (c-e) Scheme and image of GNP film preparation setup used. GNP molecules were loaded into a glass tube along with the target substrate and subsequently vacuum sealed using a mechanical pump and a flame. Then, the sealed glass tube was loaded into a furnace where it was heated to $515^{\circ} \mathrm{C}$ for $50 \mathrm{~min}$. Finally, the glass tube was opened, and various electrical and optical characterization were applied to the sample. (e) A vacuum-sealed glass tube loaded with $\mathrm{GNP} / \mathrm{C}_{96}$ and a substrate with metal electrodes. (f, g) Digital camera images of a $\mathrm{P}^{2+} \mathrm{Si} / 300 \mathrm{~nm}$ $\mathrm{SiO}_{2}$ substrate with $\mathrm{Ti} / \mathrm{Au}$ electrodes before and after sublimation of GNP. AFM images of (h) GNP and (i) $\mathrm{C}_{96}$ deposited on $\mathrm{Si} / \mathrm{SiO}_{2}$ substrate showing a flat and uniform films with RMS roughness $\sim 1 \mathrm{~nm}$. (j) AFM amplitude image showing a GNP island with multiple step edges. Inset shows an AFM height profile revealing a height of $\sim 123 \mathrm{~nm}$.

large nanographene molecules and also for the progress and development of various applications.
Here we report our finding that films of large nanographene molecules can be prepared using vacuum sublimation without 
a

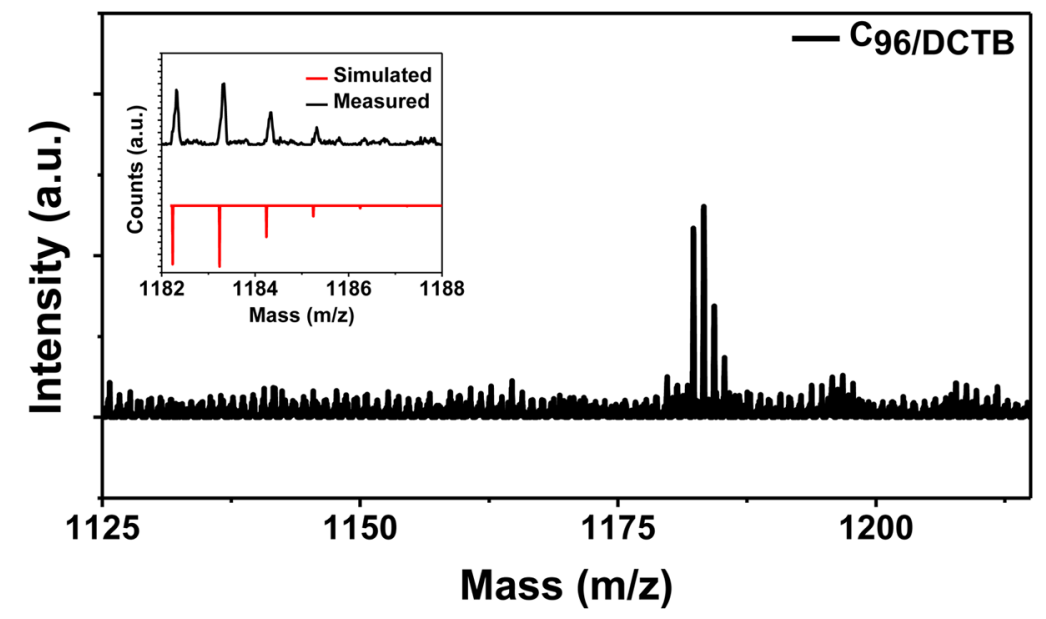

b

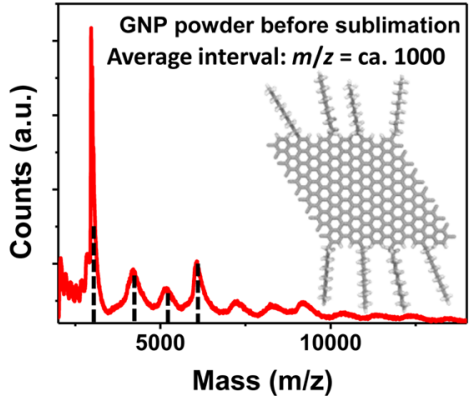

C

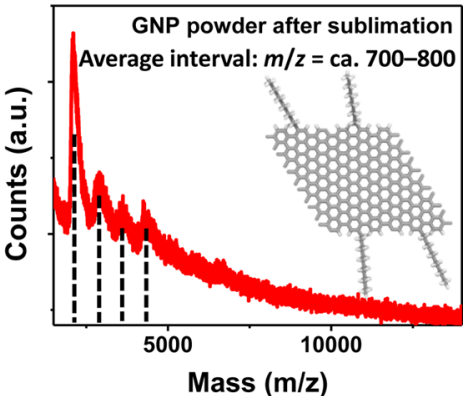

Figure 2. Investigations of the chemical composition and structure of the sublimated films. (a) Normalized MALDI-TOF MS spectrum of $\mathrm{C}_{96}$ and the reference DCTB matrix used in the spectroscopy. Inset shows the peaks in the $\mathrm{C}_{96}$ spectrum in agreement with the simulated $\mathrm{C}_{96} \mathrm{MS}$, which confirms the preservation of the molecular structure of $\mathrm{C}_{96}$ after sublimation into films. (b) MALDI-TOF MS spectrum of the synthesized GNP powder with the corresponding structure of the repeating unit shown in the inset. (c) MALDI-TOF MS spectrum of GNP powder after sublimation showing a reduced average mass interval, indicating the partial removal of alkyl chains at the edges. A suggested structure for the repeating unit after sublimation is shown in the inset (dashed black lines indicate peak positions).

destroying their aromatic core structures. Specifically, we developed a vapor-phase transport (VPT) approach, in which the large nanographene molecules are sublimated on a substrate in a vacuum-sealed glass tube. These films comprise of stacked large nanographene molecules maintaining their aromatic cores, without fusion or fragmentation. FETs based on such films exhibited current on/off ratios in the range of 140-170, which represents a significant progress compared to previously reported liquid-phase-processed large nanographene FETs., ${ }^{4,5}$ We chose two kinds of large nanographene molecules. One is $\mathrm{C}_{96}{ }^{22-24}$ bearing $96 \mathrm{sp}^{2}$ carbons in the aromatic core. This molecule has been considered to be too large for vacuum sublimation. ${ }^{22}$ Second, we chose a patch-like nanographene molecule (denoted graphene nanopatch (GNP)), which is even larger than $\mathrm{C}_{96}$ and features a uniform width of $\sim 2.1 \mathrm{~nm}$ and estimated lengths of approximately $1-10 \mathrm{~nm} .^{3}$ The GNPs employed here are shorter analogues of GNRs reported in ref 3. We chose the GNPs instead of the GNRs, since sublimation of larger GNRs is more challenging. We used atomic force microscopy (AFM), matrix-assisted laser desorption/ionization time-of-flight mass spectrometry (MALDI-TOF MS), and Raman spectroscopy to characterize the film morphology, to compare the pristine material with the sublimated films and to ensure that the deposited large nanographenes in the films maintained their basal plane structures. Moreover, we showed that GNP and $\mathrm{C}_{96}$ films can be used in the fabrication of thin-film transistors (TFT) with improved current on/off ratios by sublimating the molecules of choice onto substrates with prefabricated electrodes. Besides the observation of uniform films, we have also observed crystal-like island structures upon sublimation of GNPs, which have shown even higher electrical conductance than the films. Our VPT approach to prepare pristine nanographene molecular films suggest great promise for the future implementation of large nanograhpenes in electronic, optoelectronic, and sensing applications.

\section{DISCUSSION}

Figure 1a,b illustrates the molecular structures of GNP and $\mathrm{C}_{96}$, used in this study, respectively. Since GNPs are made through polymerization of tailor-made monomers to form corresponding polyphenylene precursors, followed by cyclodehydrogenation reaction, the GNPs, as depicted in Figure 1a, possess a certain distribution of lengths. ${ }^{3}$ By tuning the polymerization conditions, the lengths of the GNPs can be controlled. The film deposition process started with the loading of the desired amount of nanographene molecules and the target substrate in a glass tube (Figure 1c). Then, the glass tube was connected to a vacuum pump for $\sim 120 \mathrm{~s}$ and subsequently flame-sealed to keep the inside chamber in vacuum (Figure 1d). Afterward, the sealed glass tube was loaded into a furnace and was kept at $515^{\circ} \mathrm{C}$ for 50 min, where the sublimation of GNP/ $\mathrm{C}_{96}$ took place (Figure 1e). Subsequently, the furnace was slowly cooled down to room 

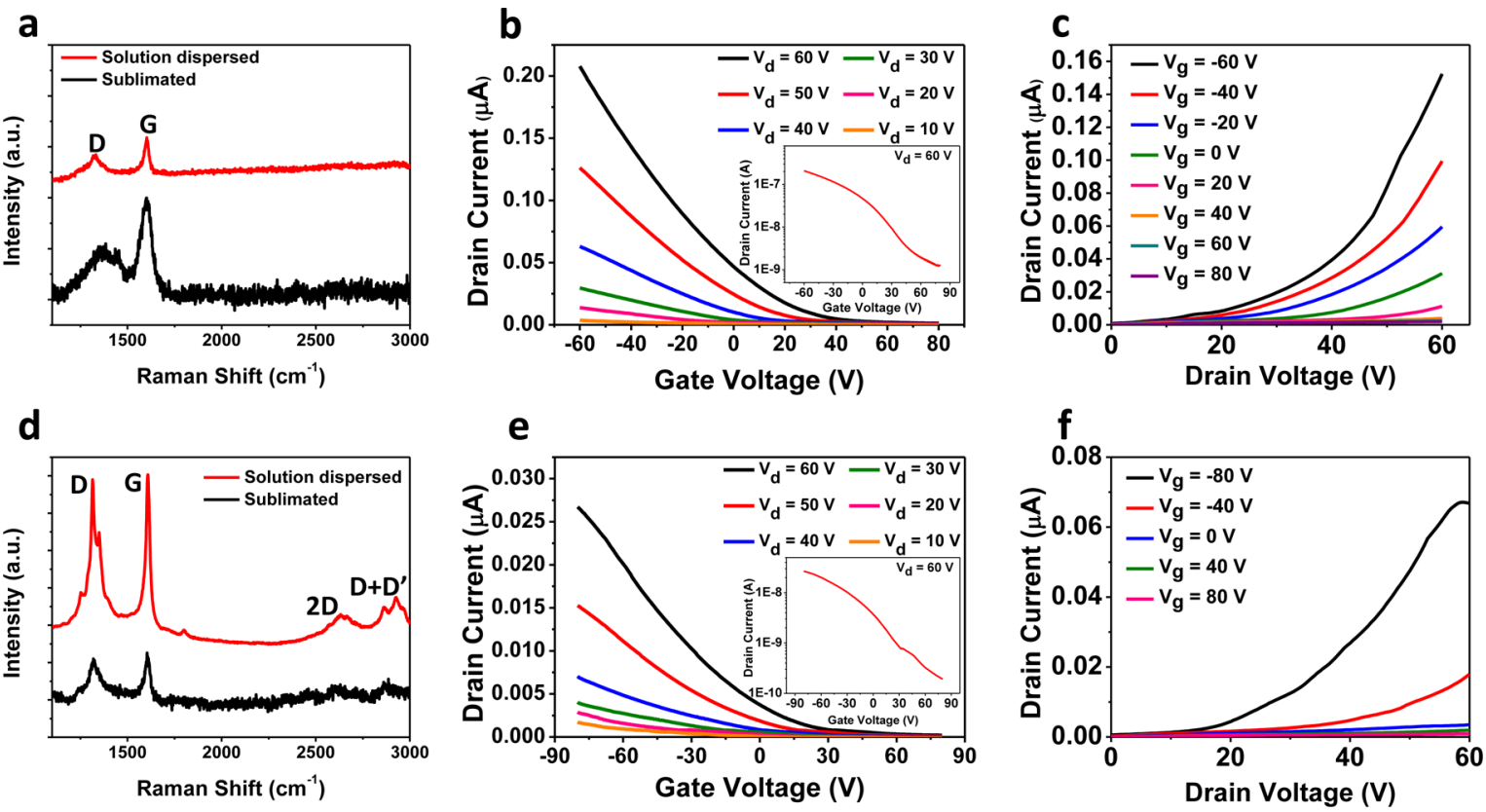

Figure 3. Raman spectra and electrical measurements of sublimated GNP/ $\mathrm{C}_{96}$ film TFTs on $\mathrm{P}^{2+} \mathrm{Si} / 300 \mathrm{~nm} \mathrm{SiO}$ substrates with Ti/Au electrodes. (a) Raman spectra of GNP films prepared by liquid-phase processing and sublimation. The spectra show G and D bands characteristic of GNP as well as qualitatively similar intensity ratios confirming the sublimation of GNP films. Laser wavelength used was $532 \mathrm{~nm}$. (b) $I_{\mathrm{d}}-V_{\mathrm{g}}$ characteristics of a GNP film TFT at different drain biases. Inset shows a logarithmic scale $I_{\mathrm{d}}-V_{\mathrm{g}}$ curve of GNP film TFT showing a current on/off ratio of $\sim 174$. (c) $I_{\mathrm{d}}-V_{\mathrm{d}}$ characteristics of the GNP film TFT at different gate voltages showing highly nonlinear curves indicating a Schottky contacted TFT. (d) Raman spectra of $\mathrm{C}_{96}$ films prepared by liquid-phase processing and sublimation. Both spectra show similar $\mathrm{G}, \mathrm{D}, 2 \mathrm{D}$, and $\mathrm{D}+\mathrm{D}^{\prime}$ bands characteristic of $\mathrm{C}_{96}$ as well as qualitatively similar G- and D-bands intensity ratios. e) $I_{\mathrm{d}}-V_{\mathrm{g}}$ characteristics of the $\mathrm{C}_{96}$ TFT at different drain biases. Inset shows a logarithmic scale $I_{\mathrm{d}}-$ $V_{\mathrm{g}}$ curve of the $\mathrm{C}_{96}$ film TFT showing a current on/off ratio of $\sim 140$. (f) $I_{\mathrm{d}}-V_{\mathrm{d}}$ characteristics of the $\mathrm{C}_{96}$ film TFT at different gate voltages showing nonlinear curves indicating a Schottky contacted TFT.

temperature in a period of $\sim 2 \mathrm{~h}$. By experimentally varying temperatures between 450 and $650^{\circ} \mathrm{C}, 515^{\circ} \mathrm{C}$ was consequently chosen as the lowest temperature that allowed formation of optically visible films. Finally, the glass tube is opened and the films are characterized. Figure 1f,g shows a $\mathrm{P}^{2+} \mathrm{Si} / 300 \mathrm{~nm} \mathrm{SiO}$ substrate with $\mathrm{Ti} / \mathrm{Au}$ electrodes before and after GNP sublimation, revealing a clear optical contrast. In addition, AFM of sublimated GNP films (Figure $1 \mathrm{~h}$ ) and sublimated $\mathrm{C}_{96}$ films (Figure 1i) showed very flat and uniform films with a rootmean-square (RMS) roughness of $\sim 1 \mathrm{~nm}$. The uniformity and smooth morphology of films is important to pursue various applications utilizing large nanographene molecules, because the rough morphology deteriorates the electronic and optical properties of the films. Interestingly, we found that under certain conditions, macro scale GNP crystal-like structures were formed after sublimation. These crystal-like structures were found to partially cover some areas of the substrate. An AFM image of a GNP crystal-like island is shown in Figure $1 j$ revealing a thickness of $\sim 123 \mathrm{~nm}$ with clearly visible step edges indicating the layered nature of the GNP crystals. This study demonstrates that VPT techniques can form crystalline structures of large nanographenes, which has not been reported before. No crystals were observed on $\mathrm{C}_{96}$ films under the sublimation conditions we tried.

An important question regarding sublimation of such large nanographenes is whether sublimation cracks and breaks the molecules into smaller fragments. Maintaining the molecular basal plane is essential to preserve the intrinsic electronic and optical properties of the molecules. To investigate this issue, we used MALDI-TOF MS to analyze the sublimated films. MALDITOF MS analysis of a sublimated film $\mathrm{C}_{96}$ showed four major peaks at masses $\sim 1182,1183,1184$, and 1185 (Figures 2a and $\mathrm{S} 1$ ), which correspond to the isotopic distribution of sublimated $\mathrm{C}_{96}$ molecules (i.e., $\mathrm{C}_{96} \mathrm{H}_{30}$ ) and are consistent with the simulated results, as shown in Figure 2a inset. This result proved that the sublimated $\mathrm{C}_{96}$ films indeed maintained their chemical identity after sublimation onto films. On the other hand, GNPs have dodecyl chains at the peripheral positions to enhance their dispersibility in organic solvents. Figure $2 b$ shows the MALDITOF MS spectrum of the GNP molecules in its pristine powder form before sublimation. Multiple peaks can be observed with an average interval of $1000 \mathrm{~g} \mathrm{~mol}^{-1}$, and the underlying reason is that the GNP molecules have a certain distribution in the number of repeating primary units due to the synthesis process. ${ }^{3}$ Figure $2 c$ shows the MALDI-TOF MS spectrum of GNP molecules after treating the GNP powder inside the glass tube at $515^{\circ} \mathrm{C}$. The MALDI-TOF MS spectrum showed a change in the average peak-to-peak mass interval from $1000 \mathrm{~g} \mathrm{~mol}^{-1}$ in the case of pristine GNP powder (Figure $2 \mathrm{~b}$ ) to $700-800 \mathrm{~g} \mathrm{~mol}^{-1}$ (Figure 2c) in the thermally treated GNP powder. The peak-to-peak interval corresponds to the mass of the primary repeating unit used as a building block to synthesize GNPs. ${ }^{3}$ It is known that the energy required to remove dodecyl side chains is much lower than the energy to break bonds in the graphene basal plane. ${ }^{25,26}$ We therefore believe the reduction in the mass of such unit at high temperature indicates the partial removal of dodecyl side chains, which is depicted in the molecular structure of the primary unit in the inset of Figure $2 \mathrm{c}$. Importantly, we believe the partial removal of dodecyl side chains is beneficial for charge transport as the dodecyl side chains might significantly degrade the conductivity of GNP films due to their insulating nature. ${ }^{20}$ 
a

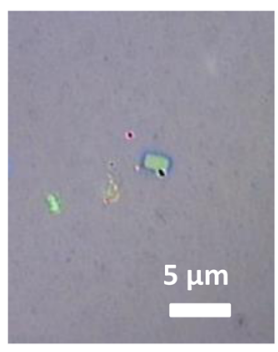

b

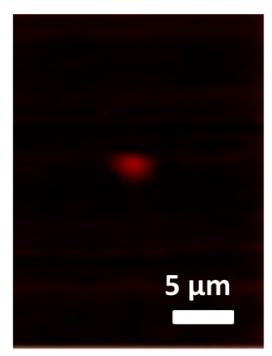

C

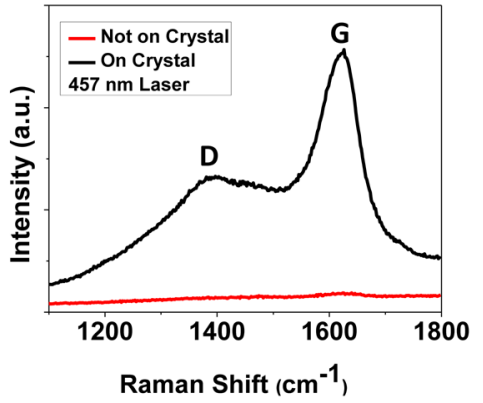

d

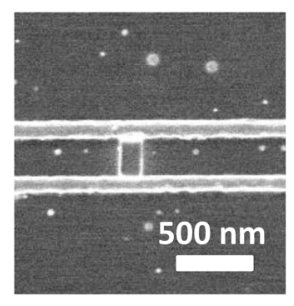

e

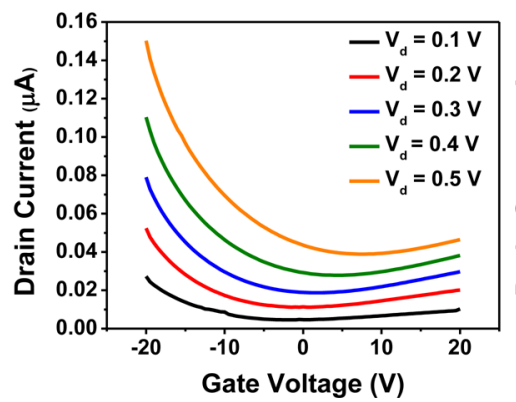

f

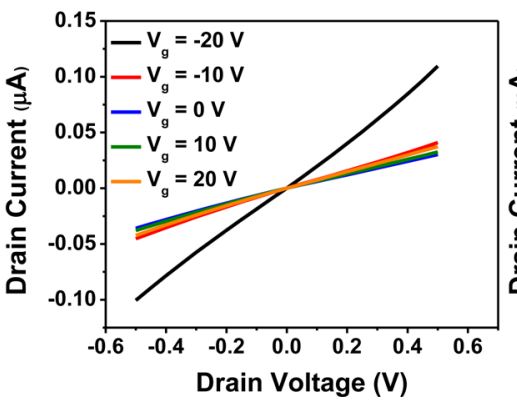

g

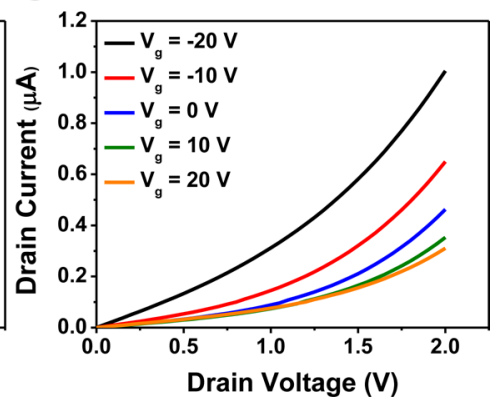

Figure 4. Characterization of GNP islands. (a) Optical image of a rectangular GNP island and (b) the corresponding Raman mapping of G band intensity using a $457 \mathrm{~nm}$ laser. (c) A typical Raman spectrum of the GNP island (black) in (a) showing G and D bands consistent with GNP films and Raman spectrum of a location away from the GNP crystal on the same substrate (red). (d) A SEM image of a GNP island bridging two Ti/Au electrodes on a $\mathrm{P}^{2+} \mathrm{Si} / 56 \mathrm{~nm} \mathrm{SiO}{ }_{2}$. (e) $I_{\mathrm{d}}-V_{\mathrm{g}}$ characteristics of a GNP crystal TFT in (d) at different drain biases. (f,g) $I_{\mathrm{d}}-V_{\mathrm{d}}$ characteristics of the GNP crystal TFT in (d) at different gate voltages. At higher values of $V_{\mathrm{d}}$ the curves appear to be slightly nonlinear indicating Schottky contacts.

One of the most promising applications for large nanographenes is TFTs. Band-like transport, which enhances the TFT device performance, was observed in highly ordered graphenebased monolayers. ${ }^{17}$ The high tunability of the electronic properties of these molecules makes them attractive for various TFT applications. However, for some recently studied chemically synthesized GNRs, the liquid-phase process of TFTs yielded nondepletable (i.e., low current on/off ratio) transistors which may be attributed to poor control over the film thickness, uniformity, and roughness due to the aggregation of the large nanographene molecules in solution. ${ }^{4,5,20}$ When the film in the channel area of a TFT has a varying thickness and/or roughness, the carrier densities in different areas of the channel would be modulated by the gate electric field differently, and thicker films may not be fully depleted of carriers by the gate electric field, thus leading to poor current on/off ratios. We have also tried preparing liquid-phase-processed GNP TFTs, which showed nonuniform film thickness, significantly large roughness $(\sim 10-$ $250 \mathrm{~nm}$ RMS roughness), and nondepletable drain current-gate voltage $\left(I_{\mathrm{d}}-V_{\mathrm{g}}\right)$ characteristics with current on/off ratios $\sim 2-3$, consistent with previous reports (Figure S2). ${ }^{4,5,20}$

In contrast, we observed that using sublimated GNP films with smooth morphologies significantly improved the current on/off ratio of GNP TFTs. We sublimated GNP films over prefabricated $\mathrm{Ti} / \mathrm{Au}$ electrodes on $\mathrm{P}^{2+} \mathrm{Si} / 300 \mathrm{~nm} \mathrm{SiO}{ }_{2}$ substrate for TFT device measurements. The sublimation of GNP films was verified by Raman spectroscopy, and the spectrum revealed similar $G$ band and D band peaks with the same G/D intensity ratios (i.e., $\sim 2$ ) for the sublimated film and the liquid-phaseprocessed films (Figure 3a), further supporting the preservation of the aromatic core structure of GNPs. The difference in the Raman spectrum signal-to-noise ratios between liquid-phase- processed films and sublimated films is because liquid-phasebased deposition led to thicker films and therefore better signalto-noise ratio than sublimation-based deposition. We have analyzed the position and the full-width at half-maxima (fwhm) of the Raman peaks for both GNPs and $\mathrm{C}_{96}$ (Table S1). First, it can be observed that the fwhm of G band (and D band) for liquid-phase-processed and sublimated GNPs are quite different while they are similar for liquid-phase-processed and sublimated $\mathrm{C}_{96}$. This could be due to the dodecyl side chain removal under high temperature in sublimated GNPs, which might induce stronger aggregations and affect the Raman peaks when compared to liquid-phase-processed GNPs, which are processed at room temperature and thus experience no side chain removal. $^{20}$ On the other hand, $\mathrm{C}_{96}$ molecules do not possess any side chains, which make the Raman spectra of the sublimated and liquid-phase-processed samples chemically identical. Figure $3 \mathrm{~b}$ shows the $I_{\mathrm{d}}-V_{\mathrm{g}}$ characteristics of the sublimated GNP TFT. Unlike liquid-phase-processed GNP TFT, the sublimated GNP films with a thickness of $\sim 2 \mathrm{~nm}$ (measured using an ellipsometer) and $\sim 3-5 \mathrm{~nm}$ (measured using an AFM) (Figure S3), showed a reasonably depletable p-type TFT with a current on/off ratio of $1.74 \times 10^{2}$ (Figure $3 \mathrm{~b}$ inset). The p-type behavior is consistent with other chemically synthesized large nanographene TFTs. ${ }^{4,5,20}$ We believe the VPT deposition played a key role for the observation of improved current on/off ratios, as VPT allowed us to produce a thin and uniform film of GNP, which ensures that the gate electric field can deplete all carriers in the channel. Because of the strong aggregation in dispersions, the films deposited from the dispersion cannot avoid the high roughness. In addition, the packing of molecules in the films is also an important factor that affects electron transport, as electrons have to move from one molecule to another before 
reaching the electrode. Figure $3 c$ shows the drain current-drain voltage $\left(I_{\mathrm{d}}-V_{\mathrm{d}}\right)$ characteristics of a GNP TFT. The nonlinearity of $I_{\mathrm{d}}-V_{\mathrm{d}}$ curves indicates a significant Schottky barrier for holes transporting from Au electrodes to GNP films. The relatively low current density (i.e., $I_{\mathrm{ON}} / W=0.2 \mathrm{nA} / \mu \mathrm{m}$ ) for GNP films might be due to both the large Schottky barrier formed at the contacts and hole transport between molecules. Nevertheless, the demonstration of an operational GNP TFT (i.e., depletable $\left.I_{\mathrm{d}}-V_{\mathrm{g}}\right)$ is an encouraging step toward the utilization of such molecules and also other large nanographenes in future electronic applications. We note that it would not be meaningful to calculate the mobility of such TFTs due to the existence of a large Schottky barrier which would significantly underestimate the intrinsic mobility of such films. We carried out TFT measurements on sublimated $\mathrm{C}_{96}$ films as well. Raman spectroscopy of both liquid-phase-processed and sublimated $\mathrm{C}_{96}$ films showed qualitatively similar spectra for both with $G / D$ ratio of $\sim 1$ (Figure 3d). Unlike GNPs, Raman spectra of $\mathrm{C}_{96}$ show $2 \mathrm{D}$ and $\mathrm{D}+\mathrm{D}^{\prime}$ peaks. Figure $3 \mathrm{e}$ shows the $I_{\mathrm{d}}-V_{\mathrm{g}}$ characteristics of the sublimated $\mathrm{C}_{96}$ TFT devices. The devices based on $\mathrm{C}_{96}$ films revealed a current on/off ratio of $1.4 \times 10^{2}$, and $I_{\mathrm{ON}} / W=0.027 \mathrm{nA} / \mu \mathrm{m}$. A significant Shottky barrier evident by highly nonlinear $I_{\mathrm{d}}-V_{\mathrm{d}}$ curve is also observed in $\mathrm{C}_{96}$ TFT devices (Figure 3f).

Another important application of GNPs is in the field of optoelectronics and photonics, due to their adjustable lengths and edge structures which directly modify their optical properties. We have studied the optical properties of GNP dispersions and films using photoluminescence (PL) and transient photoluminescence (TPL) measurements, which revealed PL peaks in both the ultraviolet (UV) and visible parts of the spectrum (Section S1).

When the GNP concentration inside the tube is sufficient, we observed that many GNP crystal-like islands grew on top of the target substrate. Figure 4a shows an optical image of the crystallike structure in Figure 1j. These crystal-like islands grew with various sizes and heights. We found that for sublimated materials with density under $0.3 \mathrm{mg} / \mathrm{cm}^{3}$, only films were formed and no islands would grow. When the sublimation starts, GNPs tend to form films covering the $\mathrm{SiO}_{2}$ surface. When the $\mathrm{SiO}_{2}$ surface is covered, GNPs start to stack up in three-dimensional (3D) structures forming the crystal-like islands. This is why the concentration of the molecule inside the chamber must be sufficient for such a process. In addition, we believe that the crystal-like islands appear to exhibit a screw-dislocation pattern, which is known as a mechanism for evolution of two-dimensional (2D) growth to 3D growth (Figure 1j). Raman mapping of the GNP crystal-like islands was carried out using a $457 \mathrm{~nm}$ laser, and the GNP island in the resultant image can be easily distinguished from the surrounding species (Figure $4 \mathrm{~b}$ ). The Raman spectrum of the crystal-like islands shows the characteristic $\mathrm{G}$ band and $\mathrm{D}$ band of GNP molecules with a G/D ratio of $\sim 2.2$ (Figure 4c), suggesting that these islands may have similar quality with pristine GNPs. In order to measure the electronic device performance of GNP islands, we fabricated prepatterned substrates with electrodes defined using electron beam lithography (EBL) with channel lengths from 100 to $500 \mathrm{~nm}$, and then GNP sublimation was carried out. Figures $4 \mathrm{~d}$ and S4 show scanning electron microscope (SEM) images of some of the observed GNP islands bridging the EBL electrodes or attached to the metal electrodes. We have carried out electrical device measurements for GNP islands bridging metal electrodes. Figure $4 \mathrm{e}$ shows the $I_{\mathrm{d}}-V_{\mathrm{g}}$ characteristics of GNP island in Figure 4d. As it can be seen, the device is nondepletable, which might be due to the thickness of the GNP island (i.e., >100 nm) causing a gate electric field screening effect. Nevertheless, the conductance of the device is greatly enhanced $\left(I_{\mathrm{ON}} / W \sim 1500 \mathrm{nA} / \mu \mathrm{m}\right.$ at $V_{\mathrm{d}}=$ $0.5 \mathrm{~V}$ ) and an effective device mobility, using the device transconductance (see Methods) in the linear region and considering all crystal-like islands bridging the electrodes, is estimated to be $\sim 1 \mathrm{~cm}^{2} / \mathrm{V} \cdot \mathrm{s}$. Figure $4 \mathrm{f}$,g shows the $I_{\mathrm{d}}-V_{\mathrm{d}}$ characteristics of GNP island devices in two different voltage ranges. The extracted mobility is underestimated because of both the Schottky barrier observed in the slightly nonlinear $I_{\mathrm{d}}-V_{\mathrm{d}}$ curve (Figure 4f,g) and also the electric field screening effect which may significantly affect the device mobility estimation as well. The growth and the measurement of GNP crystals might be an important route to create thinner crystalline GNP films for high-mobility TFTs. ${ }^{27}$ Moreover, XRD was carried out for GNP islands to investigate their structural properties. XRD revealed a peak corresponding to a lattice distance of $\sim 3.1 \AA$, whereas no obvious feature was observed for GNP flat films without islands (Figure S5b). This distinction between the XRD of GNP films and GNP islands might be either because thin GNP films (i.e., $<5$ $\mathrm{nm}$ ) are not totally crystalline due to the mismatch with the substrate or that diffraction from such thin films cannot be resolved by the XRD equipment used in the experiment (see Methods).

\section{CONCLUSION}

In summary, we have developed a method to deposit GNP and $\mathrm{C}_{96}$ films using VPT in a vacuum-sealed glass tube. Remarkably, the pristine aromatic core structure of such large nanographene molecules is preserved. AFM characterization of these films revealed smooth and flat deposited films with RMS roughness of $\sim 1 \mathrm{~nm}$ which could not be achieved using liquid-phase processing techniques. Additionally, we have characterized the sublimated molecules using MALDI-TOF MS and confirmed that the basal plane structure of large nanographene, $C_{96}$, was preserved after sublimation. This result overrides previous hypotheses and expectations that such large molecules will be subject to cracking and disintegration if thermally sublimated. Moreover, electrical device measurements of GNP and $\mathrm{C}_{96}$ based TFTs showed the creation of a depletable and operational TFT as well as the possibility of achieving relatively high mobility in FETs based on GNP crystalline samples. The results presented in this work may influence the understanding of sublimation and crystallization of such large nanographenes and could lay the foundation for future use of nanographenes in electronic and optoelectronic applications.

\section{METHODS}

Synthesis of Large Nanographenes. Both $\mathrm{C}_{96}$ and GNPs were synthesized following reported procedures. ${ }^{3,23}$ For the preparation of GNPs, polyphenylene precursors with a weight-average molecular weight of $5200 \mathrm{~g} / \mathrm{mol}$, a number-average molecular weight of $3500 \mathrm{~g} /$ mol, and a polydispersity index of 1.5 , based on size-exclusion chromatography analysis against a polystyrene standard, were used.

Sealed Glass Tube Sublimation. The $\mathrm{GNP} / \mathrm{C}_{96}$ powder was loaded along with the target substrate in a $1 / 4$ in. glass tube. Then, a mechanical pump was connected to the open end of the glass tube, which was pumped for $\sim 120 \mathrm{~s}$. Afterward, the glass tube was flamed at the side away from the powder and target substrate until that end was sealed. The tube was then loaded into a vacuum furnace and heated up to $515^{\circ} \mathrm{C}$ in $15 \mathrm{~min}$, maintained at that temperature for $\sim 50 \mathrm{~min}$, and then cooled down with the furnace cover closed until it reached room temperature $(\sim 2 \mathrm{~h})$. 
MALDI-TOF MS. Mass spectra of powder samples were recorded using a Bruker Reflex II utilizing a $337 \mathrm{~nm}$ nitrogen laser, calibrated against poly(ethylene glycol) (3000 $\left.\mathrm{g} \mathrm{mol}^{-1}\right)$, through solid-state sample preparation with tetracyanoquinodimethane as matrix. ${ }^{28}$ Mass spectrometry experiments on films were performed on a SYNAPT G2 Si instrument (Waters Corp., Manchester, UK) with matrix-assisted laser desorption/ionization (MALDI) source. For the analysis of the sublimated films, the sample wafer was fixed on the MALDI sample plate by a double-side tape. $\{(2 E)$-2-methyl-3-[4-(2-methyl-2propanyl)phenyl]-2-propen-1-ylidene $\}$ malononitrile (DCTB) matrix was subsequently sublimed on the surface of each wafer for the measurements. The mass range of 150-2000 Da was recorded with a mass resolution around 10,000.

XRD. We used Rigaku Ultima IV powder/thin-film diffractometer for the purpose of analyzing films and crystals. The sweep rate used was 0.04 $\mathrm{deg} / \mathrm{min}$, and the $\mathrm{X}$-ray generator has a $\mathrm{Cu}$ target with a maximum power output of $3 \mathrm{~kW}$.

TFT Fabrication and Measurement. Fabrication was done using regular photolithography techniques on a $\mathrm{P}^{2+} \mathrm{Si} / 300 \mathrm{~nm} \mathrm{SiO}$ substrate with $\mathrm{Ti} / \mathrm{Au}$ electrodes. Devices had a variable channel length of 3-8 $\mu \mathrm{m}$ and a channel width of $1 \mathrm{~mm}$. Device measurement was done on using an Agilent 4156B semiconductor analyzer.

UV-vis Absorption. The absorption spectrum was taken with a PerkinElmer Lambda 950 UV-vis/NIR spectrometer with an integrating sphere.

Raman Spectroscopy. Raman spectrum was taken using a Renishaw (inVia) Raman microscope with an objective lens of 100X. Laser wavelengths used were 532 and $457 \mathrm{~nm}$.

Mobility Calculation. The mobility of GNP crystal devices were calculated using $\mu=g_{\mathrm{m}} L /\left(C_{\mathrm{ox}} V_{\mathrm{ds}} W\right)$, where $C_{\mathrm{ox}}$ is the capacitance of the gate oxide per unit area, $L$ and $W$ are the channel length and width, respectively, $V_{\mathrm{ds}}$ is the drain bias, and $g_{\mathrm{m}}$ is the peak transconductance calculated from the $I_{\mathrm{d}}-V_{\mathrm{g}}$ slope.

\section{ASSOCIATED CONTENT}

\section{(S Supporting Information}

MALDI-TOF MS spectra (Figure S1); electrical measurements of liquid-phase dispersed GNP TFT (Figure S2); AFM characterization of sublimated GNP thin-film thickness (Figure S3); SEM images of GNP crystals (Figure S4); UV-vis absorption spectrum and XRD of GNP crystals and films (Figure S5); analysis of Raman spectral peaks of liquid-phaseprocessed and sublimated GNPs/ $\mathrm{C}_{96}$ (Table $\left.\mathrm{S} 1\right)$; discussion of the optical properties of GNPs (Section S1); steady-state PL spectrum of GNP dispersed in DCB (Figure S6); investigations of the optical properties of the sublimated GNP dispersion and films (Figure S7). This material is available free of charge via the Internet at http://pubs.acs.org.

\section{AUTHOR INFORMATION}

\section{Corresponding Author}

*chongwuz@usc.edu

\section{Notes}

The authors declare no competing financial interest.

\section{ACKNOWLEDGMENTS}

We acknowledge Stephen E. Bradforth and Stephen B. Cronin of University of Southern California for help with some of the experiments. We acknowledge the office of Naval Research for financial support. This research was partially supported by the European Research Council grant on NANOGRAPH, DFG Priority Program SPP 1459, Graphene Flagship (no. CNECTICT-604391), and European Union Projects UPGRADE and MoQuaS.

\section{REFERENCES}

(1) Narita, A.; Feng, X.; Hernandez, Y.; Jensen, S. r. A.; Bonn, M.; Yang, H.; Verzhbitskiy, I. A.; Casiraghi, C.; Hansen, M. R.; Koch, A. H. R.; Fytas, G.; Ivasenko, O.; Li, B.; Mali, K. S.; Balandina, T.; Mahesh, S.; De Feyter, S.; Müllen, K. Nat. Chem. 2014, 6, 126-132.

(2) Vo, T. H.; Shekhirev, M.; Kunkel, D. A.; Morton, M. D.; Berglund, E.; Kong, L.; Wilson, P. M.; Dowben, P. A.; Enders, A.; Sinitskii, A. Nat. Commun. 2014, 5, 3189.

(3) El Gemayel, M.; Narita, A.; Dossel, L. F.; Sundaram, R. S.; Kiersnowski, A.; Pisula, W.; Hansen, M. R.; Ferrari, A. C.; Orgiu, E.; Feng, X.; Mullen, K.; Samori, P. Nanoscale 2014, 6, 6301-6314.

(4) Kim, K. T.; Lee, J. W.; Jo, W. H. Macromol. Chem. Phys. 2013, 214, $2768-2773$

(5) Kim, K. T.; Jung, J. W.; Jo, W. H. Carbon 2013, 63, 202-209.

(6) Narita, A.; Verzhbitskiy, I. A.; Frederickx, W.; Mali, K. S.; Jensen, S. A.; Hansen, M. R.; Bonn, M.; De Feyter, S.; Casiraghi, C.; Feng, X.; Müllen, K. ACS Nano 2014, 8, 11622-11630.

(7) Yamamoto, Y.; Zhang, G.; Jin, W.; Fukushima, T.; Ishii, N.; Saeki, A.; Seki, S.; Tagawa, S.; Minari, T.; Tsukagoshi, K.; Aida, T. Proc. Natl. Acad. Sci. U.S.A. 2009, 106, 21051-21056.

(8) Xiao, S.; Tang, J.; Beetz, T.; Guo, X.; Tremblay, N.; Siegrist, T.; Zhu, Y.; Steigerwald, M.; Nuckolls, C. J. Am. Chem. Soc. 2006, 128, 10700-10701.

(9) Lee, J.-H.; Choi, S.-M.; Pate, B. D.; Chisholm, M. H.; Han, Y.-S. J. Mater. Chem. 2006, 16, 2785-2791.

(10) Xiao, S.; Myers, M.; Miao, Q.; Sanaur, S.; Pang, K.; Steigerwald, M. L.; Nuckolls, C. Angew. Chem. 2005, 117, 7556-7560.

(11) Pisula, W.; Feng, X.; Müllen, K. Chem. Mater. 2010, 23, 554-567.

(12) Sergeyev, S.; Pisula, W.; Geerts, Y. H. Chem. Soc. Rev. 2007, 36, $1902-1929$.

(13) Wu, J.; Pisula, W.; Müllen, K. Chem. Rev. 2007, 107, 718-747.

(14) Shklyarevskiy, I. O.; Jonkheijm, P.; Stutzmann, N.; Wasserberg, D.; Wondergem, H. J.; Christianen, P. C. M.; Schenning, A. P. H. J.; de Leeuw, D. M.; Tomović, Ž.; Wu, J.; Müllen, K.; Maan, J. C. J. Am. Chem. Soc. 2005, 127, 16233-16237.

(15) Craats, A. M. v. d.; Warman, J. M.; Fechtenkötter, A.; Brand, J. D.; Harbison, M. A.; Müllen, K. Adv. Mater. 1999, 11, 1469-1472.

(16) Tsao, H. N.; Räder, H. J.; Pisula, W.; Rouhanipour, A.; Müllen, K. Phys. Status Solidi A 2008, 205, 421-429.

(17) Käfer, D.; Bashir, A.; Dou, X.; Witte, G.; Müllen, K.; Wöll, C. Adv. Mater. 2010, 22, 384-388.

(18) Harris, K. D.; Xiao, S.; Lee, C. Y.; Strano, M. S.; Nuckolls, C.; Blanchet, G. B. J. Phys. Chem. C 2007, 111, 17947-17951.

(19) Chen, L.; Hernandez, Y.; Feng, X.; Müllen, K. Angew. Chem., Int. Ed. 2012, 51, 7640-7654.

(20) Abbas, A. N.; Liu, G.; Narita, A.; Orosco, M.; Feng, X.; Müllen, K.; Zhou, C. J. Am. Chem. Soc. 2014, 136, 7555-7558.

(21) Pisula, W.; Menon, A.; Stepputat, M.; Lieberwirth, I.; Kolb, U.; Tracz, A.; Sirringhaus, H.; Pakula, T.; Müllen, K. Adv. Mater. 2005, 17, 684-689.

(22) Rader, H. J.; Rouhanipour, A.; Talarico, A. M.; Palermo, V.; Samori, P.; Müllen, K. Nat. Mater. 2006, 5, 276-280.

(23) Iyer, V. S.; Wehmeier, M.; Brand, J. D.; Keegstra, M. A.; Müllen, K. Angew. Chem., Int. Ed. 1997, 36, 1604-1607.

(24) Tomović, Ž.; Watson, M. D.; Müllen, K. Angew. Chem., Int. Ed. 2004, 43, 755-758.

(25) CRC Handbook of Chemistry and Physics; Lide, D. R., Ed.; Taylor and Francis Group: Boca Raton, FL, 2006; Vol. 87.

(26) Müller, M.; Kübel, C.; Müllen, K. Chem.—Eur. J. 1998, 4, 20992109.

(27) Yuan, Y.; Giri, G.; Ayzner, A. L.; Zoombelt, A. P.; Mannsfeld, S. C. B.; Chen, J.; Nordlund, D.; Toney, M. F.; Huang, J.; Bao, Z. Nat. Commun. 2014, 5, 3005.

(28) Przybilla, L.; Brand, J.-D.; Yoshimura, K.; Räder, H. J.; Müllen, K. Anal. Chem. 2000, 72, 4591-4597. 